\title{
Tagungsberichte
}

\section{Stand der Waldschadensforschung in Bayern}

\section{- 1. Statusseminar in Neuherberg/München, 27. 02. -01.03. 1989}

Die Bestätigung bestimmter Hypothesen über die Ursachen der neuartigen Waldschäden, aber auch neue Fragen und Überraschungen brachte das Statusseminar zur Waldschadensforschung, das die „Projektgruppe Bayern zur Erforschung der Wirkung von Umweltschadstoffen " bei der Gesellschaft für Strahlen- und Umweltforschung im Auftrag des bayerischen Umweltministeriums durchgeführt hat.

Grundsätzlich, so das Umweltministerium, zeigen die Ergebnisse der bisherigen Forschungen, daß für die Waldschäden ein Komplex verschiedener Einflußfaktoren maßgeblich ist, die regional unterschiedlich wirksam sind. Eine wesentliche Rolle spielen dabei Luftverunreinigungen, die aber im Zusammenhang mit standortspezifischen Faktoren wie Witterung, Bodenverhältnisse und unterschiedliche Empfindlichkeit der Bäume gesehen werden müssen.

Die Waldschäden in höheren Lagen der Mittelgebirge scheinen auf eine durch saure Niederschläge ausgelöste Nährstoffverarmung des Bodens zurückzuführen zu sein. Andererseits tritt durch Eintrag von stickstoffhaltigen Verbindungen ein „Dünge-Effekt" hinzu, der die Nährstoffungleichgewichte weiter verschärft. Diese Hypothese hat sich für das Fichtelgebirge erhärtet, dürfte aber auch für den Bayerischen Wald gelten. Auch für die Waldschäden im bayerischen Alpenraum hat die Wirkung von Luftverunreinigungen auf den Boden gegenüber der Hypothese direkter Wirkungen von Photooxidantien, wie z.B. Ozon auf oberirdische Pflanzenteile, an Bedeutung gewon- nen*. Keine Bestätigung fand dagegen die Annahme biotischer und klimatischer Faktoren als primäre Ursachen der Waldschäden.

Die Tagung lieferte auch eine Reihe unerwarteter Ergebnisse:

- Im „Höglwald“ bei Augsburg zeigen die Bäume trotz jahrelanger saurer Beregnung des Bodens eine unverändert hohe Vitalität. Pflanzenphysiologische Untersuchungen ergaben eine überraschend hohe Säuretoleranz und Anpassungsfähigkeit des pflanzlichen Entgiftungsstoffwechsels.

- Neu war auch, daß im sauren Nebel Nitrophenole, die durch atmosphärisch-chemische Prozesse aus Komponenten der Autoabgase entstehen, in beachtlichen Konzentrationen auftreten.

Nach übereinstimmender Meinung besteht nach wie vor erheblicher Bedarf an Grundlagenforschung. So müssen die Bedeutung organischer Stoffe - u.a. auch von Pflanzenschutzmitteln als Schadfaktoren - sowie Fragen der Pflanzenernährung und des Entgiftungsstoffwechsels geklärt werden. In der Freilandforschung sind vor allem im Alpenraum atmosphärische Untersuchungen, Immissionsund Depositionsmessungen sowie verstärkte pflanzenphysiologische und Bodenuntersuchungen notwendig. Für die erst später aufgetretenen Schäden an Laubbäumen muß die Ursachenforschung erheblich intensiviert werden.

*Vgl. Heft 1 UWSF-Z. Umweltchem. Okotox., S. 55

\section{Physik und Chemie atmosphärischer Immissionen}

\section{- 10. Symposium „Naturwissenschaftliche Umweltprobleme“, Leipzig, 17./18. 01. 1989}

In Sektion 1 wurden Beiträge zur Entwicklung von Meßmethoden und -geräten zusammengefaßt, in Sektion 2 Untersuchungen anthropogener Eingriffe in Chemie und Physik der Atmosphäre. Ziel der Tagung war es, Erkenntnisfortschritte, Entwicklungstendenzen und Untersuchungsmethoden auf diesem wichtigen Teil- gebiet der Umweltwissenschafren zu diskutieren. Veranstalter: Präsidium der Kammer der Technik der DDR (Kommission Umweltschutz, AG(Z) Reinhaltung der Luft) und die Karl-Marx-Universität Leipzig.

\section{Altlastensanierung}

\section{- Workshop „Remedial Action at Abandoned Waste Sites“, Bonn, 13./14. 10. 1988}

Die Zusammenarbeit zwischen dem Bundesminister für Umwelt, Naturschutz und Reaktorsicherheit und der Umweltbehörde der USA (U.S. Environmental Protection Agency, US-EPA) zur Bewältigung der Altlastenproblematik begann am 13./14. Oktober 1988 mit einem zweitägigen Workshop. Auf Einladung des Bundesumweltministers und des Umweltbundesamtes informierten sieben $\mathrm{Ex}$ perten der EPA über die amerikanischen Strategien zur Bewältigung der Altlastenproblematik.

Das Problem der Altlasten stellt sich ausnahmslos allen industrialisierten Ländern, und seine (auch finanzielle) Lösung erfordert die Kooperation zwischen Staat, Wirtschaft und Wissenschaft. In der Bundesrepublik Deutschland gibt es eine Erfassung von Altlasten auf Länderebene seit Ende der 60er Jahre, die dann mit dem Abfallbeseitigungsgesetz von 1972 eine gesetzliche Grundlage auf Bundesebene erhielt. Man rechnet z.Zt. mit ca. 42.000 Verdachtsflächen im Bereich von Altablagerungen und kontaminierten Industriestandorten (TOPFER). Die Kommunen erstellen Kataster der Verdachtsflächen; einheitliche Bewertungsschemata existieren bisher nicht. Es ist daher notwendig, Kriterien für eine Prioritätenliste entsprechend der Sanierungsnotwendigkeiten auszuarbeiten. Nach durchgeführter Sanierung ist eine Überwachung erforderlich.
Im Mittelpunkt des Workshop stand der amerikanische Ansatz zur Altlastensanicrung auf der Grundlage des "Superfund-Gesetzes“ aus dem Jahr 1986 (SARA = Superfund Amendments and Reauthorization Act). Je nach Dringlichkeit der Sanierungsbedürftigkeit werden Removal Actions (Sofortmaßnahmen; weniger als $1 \mathrm{Jahr}$, Kosten unter US \$ 2 Mio, z.B. Ausheben) oder Remedial Actions (Sanierungsmaßnahmen; länger als $1 \mathrm{Jahr}$ erforderlich) beschlossen. Das "Hazardous Ranking System" (HRS) ermittelt die akuten oder potentiellen Gefahren, die von Chemikalien in einem kontaminierten Gebiet ausgehen. Auf der Basis des HRS entscheidet sich durch Anwendung einer Punktwertung, ob eine Altlast auf der nationalen Prioritätenliste (NPL = National Priority List) erscheint oder nicht. Betrachtet werden die Ausbreitungspfade einer Kontamination über den Grundwasserpfad, Oberflächenwasserpfad und Luftpfad. Zur Zeit sind ca. 1.200 Standorte auf der NPL. Die Sanierung der sog. "Superfund-Sites" wird aus Mitteln der EPA finanziert, wenn es nicht gelingt, den Verursacher der Kontamination zu ermitteln.

Dr. Heidelore Fiedler Universität Bayreuth 